\title{
Selection Algorithm of Virtual Enterprise Partner Based on Task- Resource Assignment Graph
}

\author{
Xue $\mathrm{Jia}^{1}$ \\ 1. Department of health management, Xi'an Medical University Xi'an Shaanxi, \\ 710021 China \\ E-mail:87149272@qq.com
}

\begin{abstract}
The project deployment diagram, which is used to uniformly describe the process and resource of virtual enterprise is established aiming at how to choose virtual enterprise partner of time-cost tradeoff and set task-resource assignment graph as scheduling model, the iterative heuristic algorithm is adopted based on relative cost-effectiveness to solve. The production of virtual partner and transportation time and cost are taken into account in algorithm, which may ensure deadline constraint as well as effectively cut down overall cost. Such algorithm applying to resource distribution and a vast amount of simulative tests proved that this method is able to effectively cut down the operation time of obtaining prioritization scheme.
\end{abstract}

Keywords: Virtual enterprise; Partner selection; Project deployment diagram; Taskresource assignment graph; Relative cost-effectiveness

\section{Introduction}

Virtual enterprise is the major mode of carrying through production operation and market competition for 21th century enterprise, more attention is being paid to it due to strengths such as lower cost, quick response to market opportunities and favorable adaptability etc. The key to success of virtual enterprise lies in how to select the most appropriate enterprise to product from heaps of potential collaborators whose are provided with diversified service quality (for example, cost factors, productive time, production quality, trust cooperation and risk factors etc). Aiming at the deficiency of partner optimization algorithm based on process model analysis and graph theory thought, this article put forward a kind of newly inspirational partner optimization algorithm based on the thought of graph theory and relative cost-effectiveness [11]. The project deployment diagram is at first established by algorithm to uniformly describe process model and resource model of enterprise and select a task-resource assignment graph [12] randomly from project configuration graph as a preliminary solution, adjust cooperative partners gradually within closing date, get the partners constantly with the maximum cost reduction inside and kick out those with maximum growth of time cost. Each adjustment of algorithm is from a global perspective with the consideration of process time and cost of cooperative partners and transportation time and cost of workpiece, which may reasonably optimize overall cost as well as avoid resource conflict of candidate partners. The simulate result proved performance and efficiency of algorithm. 


\section{Problem Description}

The project of virtual enterprise can be denoted with two-tuples $\langle D A G, R\rangle$, of which DAG (Directed Acyclic Graph) is used to describe process model, DAG $=\langle\mathrm{V}, \mathrm{L}\rangle$, $\mathrm{V}=\left\{\mathrm{v}_{i} \mid 1 \leq \mathrm{i} \leq \mathrm{n}\right\}$ are subproject collection with mutual independence and some logical relation. $\mathrm{L}=\left\{\mathrm{l}_{\mathrm{ij}} \mid \mathrm{i} \geq 1, \mathrm{j} \leq \mathrm{n}, \mathrm{i} \neq \mathrm{j}\right\}$ is the set of subproject correlation, $\mathrm{l}_{\mathrm{ij}}=\left(v_{i}, v_{j}\right)$ represents sequential relationship between $v_{i}$ and $v_{j}$ on execution, namely subproject $v_{j}$ is not allowed to get it started until $v_{i}$ have the execution completed, when $v_{i}$ is precedence subproject to $v_{j}$, and $v_{j}$ is successor subproject to $v_{i} . R=<E, D>$ is used to describe resource model, $\mathrm{E}=\bigcup_{i}^{n} \mathrm{E}_{i}=\bigcup_{i}^{n}\left\{\mathrm{e}_{i u} \mid 1 \leq u \leq \mathrm{m}_{i}\right\}$ is the set of candidate enterprise, $m=\left|\bigcup_{i} E_{i}\right|$ is the total number of virtual enterprise partners, “ || " is the potential operator of set; the candidate enterprise set of subproject $v_{i}$ is $E_{i}, m_{i}$ is the number of candidate enterprises for subproject $v_{i}, e_{i u}=\left(c_{i u}, t_{i u}\right)$ represents QoS attribute description of candidate enterprise $u$ of subproject $v_{i}$, this article primarily focuses on finished cost $c_{i k}$ and time $t_{i k} . D=\left\{d_{i u, j v} \mid 1 \leq i, j \leq n, 1 \leq u \leq m_{i}, 1 \leq v \leq m_{j}, l_{i j} \in L\right\}, \quad d_{i u, j v}=\left(c_{i u, j v}, t_{i u, j v}\right)$ represents the cost and time of transportation for workpiece between two partners when $v_{i}$ is being processed in partner enterprise $u$ and when $v_{j}$ is being processed in partner enterprise $v$. This article assumed that there has no constraints for processing capability of each virtual enterprise partner, however, there's only one subproject is able to process at random time, and there does exist transmission line between two random partners with no restraints of transmission capability, however, there's only one workpiece is able to transport at random time.

The target of partner selection is to select a candidate partner for each subproject so that the project can be completed at the lowest cost within closing time, the formal description of problem as follows:

$$
\begin{gathered}
M_{u, v}\left(\sum_{i} x_{i u} c_{i u}+\sum_{i} \sum_{j} x_{i u} x_{j v} c_{i u, j v}\right) \quad 1 \leq i, j \leq n, 1 \leq u \leq m_{i}, 1 \leq v \leq m_{j} \\
\text { s.t. } \sum_{u=1}^{m_{i}} x_{i u}=1 \quad 1 \leq \mathrm{i} \leq n \\
f_{i u t}+x_{i u} \mathrm{t}_{i u}+x_{i u} x_{j v} \mathrm{t}_{i u, j v} \leq \mathrm{f}_{j v} \quad 1_{i j} \in L, 1 \leq i, j \leq n, 1 \leq u \leq m_{i}, 1 \leq v \leq m_{j} \\
\left(f_{i u}-x_{i u} t_{i u}-x_{i u} x_{j u} \mathrm{t}_{i u, j u}-f_{j u}\right) *\left(f_{j u}-x_{j u} t_{j u}-x_{j u} x_{i u t} \mathrm{t}_{j u, i u}-f_{i u}\right) \geq 0 \quad 1 \leq u \leq m_{i}, 1 \leq i, j \leq n \\
f_{0 u}=0, f_{u u}=\mathrm{T} \\
x_{i u}=\{0,1\}, \quad 0 \leq \mathrm{i} \leq n, 1 \leq u \leq m_{i}
\end{gathered}
$$

Where, the value of $x_{i u}$ represents if subproject $v_{i}$ is processed by partner $u$; $f_{i u}$ represents completion time of subproject $v_{i}$ in partner $u$. formula (1) is the cost to partner selection scheme and target to optimization problem; formula (2) represents each subproject can be only processed by one partner; formula (3) represents meeting the demands of subproject process for partial-order constraint and completion time for transportation; formula (4) is constraints of sharing resource, which means any partner is forbid to process multi-subprojects at any time; formula (5) is the date of completion for subproject and formula (6) explains that $x_{i u}$ is Boolean variable. 


\section{Selection Algorithm of Virtual Enterprise Partner}

\subsection{Related Definition}

To intuitively reflect processing capability of virtual partner to different subprojects, transportation performance and possible conflict of sharing resource, for one thing, the process model and resource model of virtual enterprise project shall be transformed into project deployment diagram.

Definition 1. Figure $\mathrm{DAG}=<\mathrm{V}, \mathrm{L}>$ is given, the depth $B D(i)$ of subproject $v_{i}$ is defined as the longest path length (the path length means the number of edge) from $v_{i}$ to exit knot $v_{n}$.

$$
B D(i)=\left\{\begin{array}{cc}
1 & \mathrm{i}=1 \\
\max _{j \in \text { succ }(i)}\{B D(j)+1\} & \text { otherwise }
\end{array}\right.
$$

Definition 2 The project deployment diagram $G=\left\langle V^{\prime}, L^{\prime}, E^{\prime}, D^{\prime}\right\rangle$ of virtual enterprise project $<D A G, \mathrm{R}>$ is a multilayer network diagram of $B D(n)$, the number of network node in $k$ layer $n_{k}=\left\{\bigcup_{j} E_{j} \mid B D(j)=k\right\}$; if there exist identical node in adjacent layer back and forth, the transmission time and cost of workpiece between them both are 0 ; if $d_{i u, j v} \in D\left(l_{i j} \in L, D B(i)=k, D B(j)=k+1\right\}$, there does exist networking between $u$ node of $k$ layer and $v$ node of $k+1$ layer, processing time of subproject $i, j$ on $u, v$ node are $c_{i u} c_{j v}$ and $t_{i u}, t_{j v}$, respectively, transportation cost and time are $c_{i u, j v}$ and $t_{i u, j v}$ respectively.

The project employment diagram may uniformly describe the processing performance of candidate enterprise partner for virtual enterprise projects and subprojects and transportation performance of workpiece between candidate enterprise partners.

Definition 3 TRAG (Task-Resource Assignment Graph) is a subgraph that meets with DAG and resource constraints on project deployment diagram, each TRAG is a solution to selection problem of virtual enterprise partner. If each task selects virtual partner with shortest processing time, and transportation time is assumed as 0 , then the critical path of such scheme is MCP (Minimum Critical Path), and the path length of it is called as minimum fastest time.

Definition 4 Suppose $u_{1}$ and $u_{2}$ are optional two network nodes of $j_{1}$ subproject on $j$ layer, respectively, precedence $i_{1}$ and successor $k_{1}$ are $k_{q}$ of $j_{1}$ subproject in TRAG are undertaken by network node $i_{p}$ on $i$ layer and $k_{q}$ on $k$ layer, respectively, network node $i_{p}$ on $i$ layer and $u_{1} \& u_{2}$ on $j$ layer both are provided with direct network connection, as well as $u_{1} \& u_{2}$ on $j$ layer and $k_{q}$ on $k$ layer are also provided with network connection. The task-resource assignment graph transformed into TRAG' when moving $j_{1}$ subproject to $u_{2}$ node from $u_{1}$ node, then relative cost effectiveness $r\left(j_{1},\left(u_{1}, u_{2}\right)\right)$ is ration between overall cost and completion time transformation after TRAG is being varied, that is:

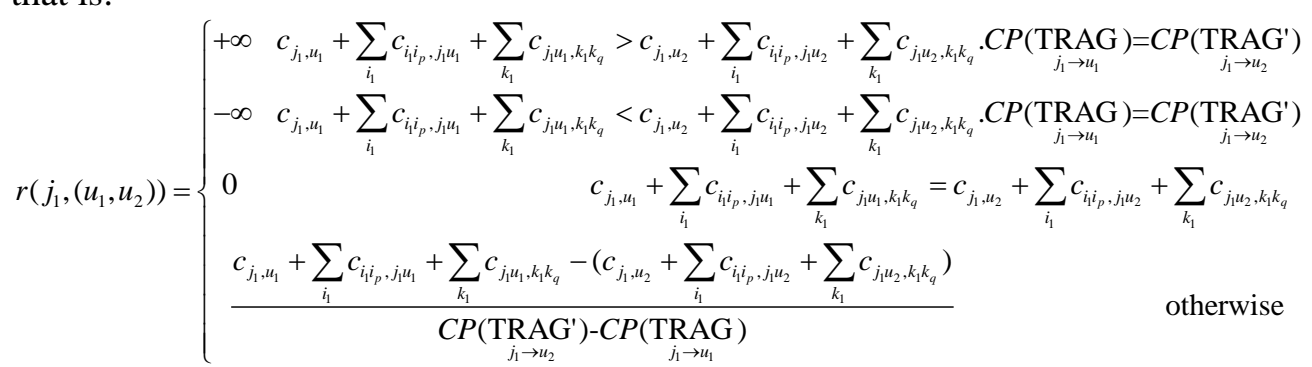


Where, $C P$ (TRAG) represents critical path length of TRAG when submission $j$ is being assigned to partner $u$.

Relative cost-effectiveness $r\left(j_{1},\left(u_{1}, u_{2}\right)\right)$ showed that the variation of cost time to scheduling plan when subproject $j_{1}$ is transforming from $u_{1}$ network node on $j$ layer to $u_{2}$ node under the condition that any other subprojects in the whole project remain unchanged. $r\left(j_{1},\left(u_{1}, u_{2}\right)\right)<0$, overall time and overall cost variation, which generated from variation of processing node, are identical, with the selection of candidate partners with lead-time reduction may synchronously decrease overall cost. When $r\left(j_{1},\left(u_{1}, u_{2}\right)\right)=-\infty$, the change of candidate partner merely make overall cost increased but critical path length remains the same; $r\left(j_{1},\left(u_{1}, u_{2}\right)\right)>0$, the variation of critical path cost and overall cost, which generated from variation of processing node, are diversified, with the selection of candidate partner who will help increase critical path length may cut down the cost, yet with the selection of candidate partner with increased cost may reduce time, and with greater relative cost-effectiveness comes with an significant result. When $r\left(j_{1},\left(u_{1}, u_{2}\right)\right)=\infty$, the change of candidate partner will lead to a decrease of overall cost, but overall time remains unchanged.

\subsection{Algorithm Description}

Algorithm 1 Conversion algorithm from DAG to project deployment diagram Input: DAG, matrix E and D

Output: project deployment diagram

(1) Calculate the depth of each subproject in DAG according to formula (7);

Determine an overall layers of project deployment diagram according to $B D(n)$;

(2) Determine virtual enterprise partners on each layer of project deployment diagram according to matrix E;

(3) Add network connection to identical node of adjacent layers back and forth;

According to matrix D, if $d_{i u, j v} \in D\left(l_{i j} \in L, D B(i)=k, D B(j)=k+1\right\}$, network connection is created between $u$ node on $k$ layer and $v$ node on $k+1$ layer;

(4) Output project deployment diagram;

Algorithm 2 Partner selection algorithm based on figure and relative cost-effectiveness Input: project deployment diagram $\mathrm{G}$ and deadline $\mathrm{T}$ given by users

Output: selection scheme of virtual partner

(1) Compute relative cost-effectiveness of project deployment diagram G;

$k_{0}=0$; a task-resource assignment graph is generated randomly as an initiatory partner selection scheme and compute critical path time $T_{s}$ of task-resource assignment graph;

Select subprojects of virtual enterprise whose relative cost-effectiveness is negative and critical path length is reduced, or relative cost-effectiveness is $\infty$ on task-resource distribution diagram, compute the critical path time $T_{s}$ of task-resource assignment graph by adjusting scheduling plan;

(4) If $T_{s}>T$, there are subproject partners whose are provided with maximum relative cost-effectiveness with positive value and a decreased critical path length in adjustive task-resource assignment graph; if $T_{s} \leq T$, there are subproject partners whose are provided with maximum relative cost-effectiveness with positive value and an increased critical path length in adjustive task-resource assignment graph. If there appears to be cyclic sequence in the sequence of relative cost-effectiveness with most-positive value in schedule, switch it to step (5), otherwise switch it to step (3).

(5) Select task-resource assignment graph whose completion time is lower than and next to deadline as an optimal partner selection scheme and input. 
Property 1 The scheduling method of relative cost-effectiveness is convergent.

Prove: without loss of generality, suppose time cost of different scheduling schemes of some subproject on critical path of virtual enterprise as shown in figure 1.

Suppose that there has no virtual partners whose relative cost-effectiveness is positive and critical path is reduced, or its relative cost-effectiveness is $\infty$ in other subprojects of scheduling scheme, all of relative cost-effectiveness with positive value are less than $\mathrm{r}$, $\mathrm{r} 2, \mathrm{r} 3$ and $\mathrm{r} 4, \mathrm{t} 1$ is the process of $\mathrm{j}$ and time limit of workpiece transmission of subproject, partner 1 is the current selection of subproject $\mathrm{j}$, the completion time of scheme exceeds deadline by this moment and the relative cost-effectiveness $r(j,(1,2)), r(j,(1,5))$ and $r(j,(1,4))$ under scheduling scheme are less than 0 and $r(j,(1,2))$ being the minimum, $r(j,(1,6)), r(j,(1,7))$ and $r(j,(1,8))$ are greater than 0 .

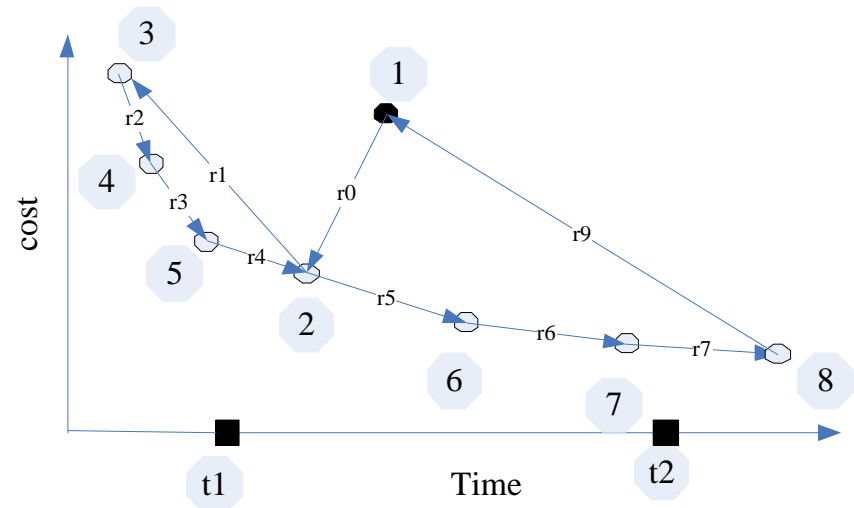

Figure 1. Time Cost of Different Scheduling Scheme of Some Subproject in Virtual Enterprise

\subsection{Examples}

Some virtual enterprise is composed of 6 subprojects whose sequential relationship is shown as in figure 2. Each subproject is bid by candidate enterprises of 3、2、3、3、3 、 2 in six candidate enterprises, respectively. Processability and transport properties of these candidate enterprises are described with table 1 and table 2, respectively. The deadline given by users is 18 .

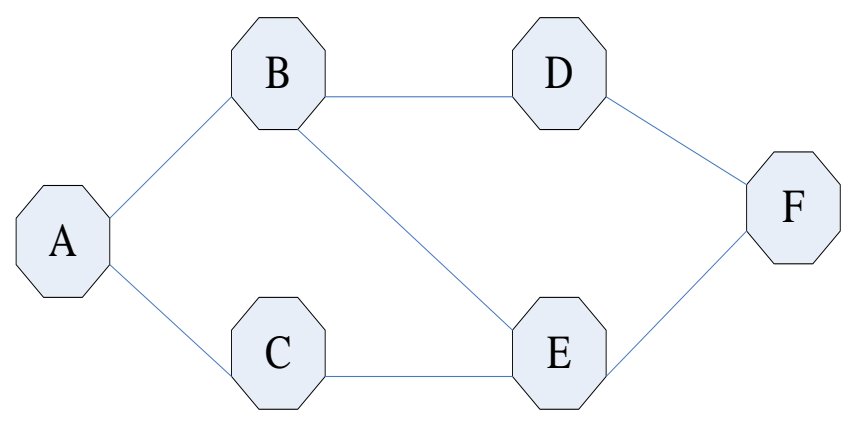

Figure 2. DAG Graph of Virtual Enterprise Project 
Table 1. E Matrix of Virtual Enterprise Project

$$
\left[\begin{array}{cccccc}
1 & 2 & 3 & 4 & 5 & 6 \\
(10,5) & - & - & (8,4) & - & (9,4) \\
- & (4,2) & (8,2) & - & - & - \\
- & - & (18,4) & - & (4,2) & (5,2) \\
(25,5) & - & (20,6) & - & - & (33,6) \\
(11,3) & - & - & - & (12,3) & (9,3) \\
- & (10,3) & (13,3) & - & - & -
\end{array}\right]
$$

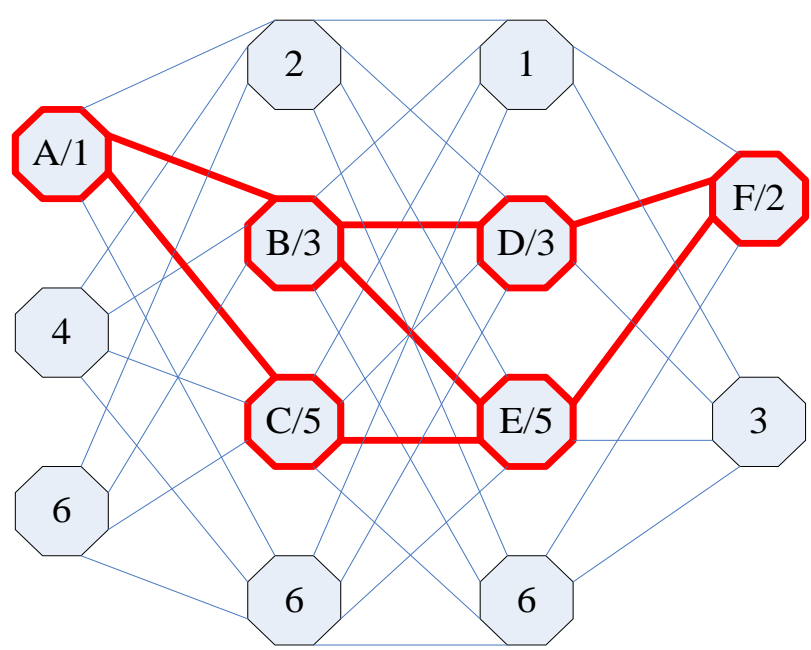

Figure 3. Project Deployment Diagram of Virtual Enterprise and Initial TaskResource Assignment Graph

Suppose that initial task-resource assignment graph of virtual enterprise, as shown in figure 3, then overall cost and time are 76 and 20, respectively, the relative costeffectiveness of subproject A $r(A,(1,4))=-4, r(A,(1,6))=-\infty$, owing to the existing time of this subproject and candidate partner 4 of relative cost-effectiveness with negative value, select the partner of subproject A as 4 . An overall cost and time of task-resource assignment graph are 72 and 19, respectively, as shown in figure 4(a).

The relative cost-effectiveness of subproject $\mathrm{C}$ is $r(C,(5,3))=-6$, however, but the time for partner 3 is increased; $r(C,(5,6))=-2.5$, but the time for partner 6 is increased, hence making no adjustments to partner of subproject $C$; the relative cost-effectiveness of $\mathrm{B}$ is $r(B,(3,2))=2$, where has no candidate partners of relative cost-effectiveness with negative value, the partner of subproject $B$ remains unchanged; the relative costeffectiveness of subproject $\mathrm{D}$ is $r(D,(3,1))=r(D,(3,6))=-\infty$, on account of an increased cost, hence the partner of subproject $\mathrm{D}$ remains unchanged as well; the relative costeffective of subproject $\mathrm{F}$ is $r(F,(2,3))=0.5$, where has no candidate partners of relative cost-effectiveness with negative value and decreased critical path length or of infinity positive value, the partner of subproject $F$ still remains unchanged. 


\section{Experimental Result and Analysis}

Simulation test is adopted to virtual enterprise applications of diversified DAG to assess the performance of estimate method and make it compare with performance and efficiency of branch and bound method. The experimental simulation environment is PIV 2.6GH, 512MB with Windows2000 operating system. DAG auto-creator of documentsimilar [11] is introduced in process model of virtual enterprise, and the number of node will be inputted by users. Fixed value 5 is applied to the number of virtual partner. Finished $\operatorname{cost} c_{i k}$ and time $t_{i k}$ of subproject $v_{i}$ in candidate enterprises are random number of 20 and 10 for seed, respectively; transportation $\operatorname{cost} c_{i u, j v}$ and time $t_{i u, j v}$ of workpiece between two partners are random number of 10 and 5, respectively, for seed when subproject $v_{i}$ is being processed in partner enterprise $u$ and $v_{j}$ in partner enterprise $v$. Take $2 *$ MCP as completion time given. DAG $(10,15,20,25$ and 30, respectively) of each node number runs 10 times, respectively, that is, make a comparison of average cost and operation time.

The expense cost and operation time of ultimate scheduling scheme for two algorithm on different nodes are described in figure 4. The dot line is results from proposed algorithm, an optimal cost and operation time of DAG node, from high to low is: 10, 15, 20, 25 and 30; the border line results from branch and bound method, an optimal cost and operation time of DAG node, from high to low is: 10, 15, 20, 25 and 30.

Comparative effectiveness of two algorithms is shown in figure: (1) as an increase of the number of DAG node, computing time of proposed algorithm has a linear increasing trend, however, the computing method of branch and bound method has an index increasing trend. This is because proposed algorithm all the time selects virtual partners whose processing time is being the lowest when completion time is in excess of deadline, the other way around, and original partner of corresponding task will be replaced. (2) For DAG with identical number of node, final cost obtained from brand and bound method is superior to proposed algorithm, however, the difference between them will get smaller and smaller as the number of node increases. This is because there exist a time fragment between completion time of final scheme and given deadline in proposed algorithm under different DAG and it fails to remedy with cost, as an increase of the number of DAG node, this unoccupied time fragment will become small and the effect results from cost remedy will be lower and lower.

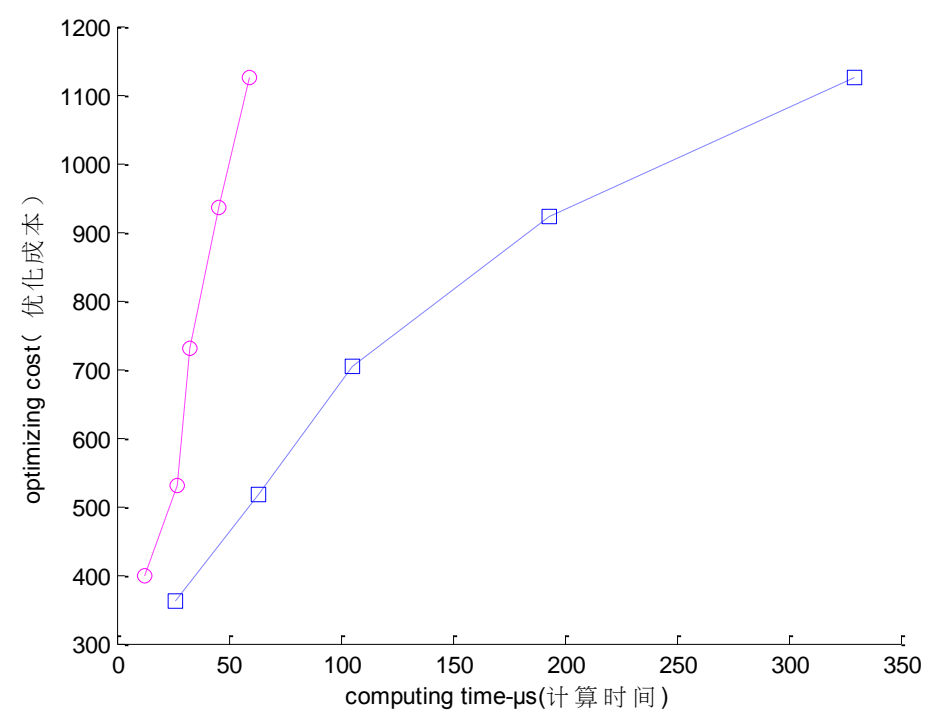

Figure 4. Computing Time and Cost Expenses of Final Scheme Obtained from Two Algorithms 


\section{Conclusion}

Virtual enterprise partner selection with transportation time and cost of workpiece is a typical NP-Hard problem. This article established project deployment diagram model from optimal selection of virtual enterprise partner and set task-resource assignment graph as solution scheme, heuristic algorithm, which is used to gradually adjust task-resource assignment graph within given deadline in the process of partner optimization to realize optimization solution, based on relative cost-effectiveness is given. Effectiveness of model and its solution algorithm are certified using examples.

\section{References}

[1] Lv Z, Tek A, Da Silva F. Game on, science-how video game technology may help biologists tackle visualization challenges[J]. PloS one, (2013), 8(3): 57990.

[2] Su T, Wang W, Lv Z. Rapid Delaunay triangulation for randomly distributed point cloud data using adaptive Hilbert curve[J]. Computers \& Graphics, (2016), 54: 65-74.

[3] Jinyu Hu, Zhiwei Gao and Weisen Pan. Multiangle Social Network Recommendation Algorithms and Similarity Network Evaluation[J]. Journal of Applied Mathematics, 2013 (2013).

[4] Gu W, Lv Z, Hao M. Change detection method for remote sensing images based on an improved Markov random field[J]. Multimedia Tools and Applications, (2015): 1-16.

[5] Chen Z, Huang W, Lv Z. Towards a face recognition method based on uncorrelated discriminant sparse preserving projection[J]. Multimedia Tools and Applications, (2015): 1-15.

[6] Jinyu Hu and Zhiwei Gao. Distinction immune genes of hepatitis-induced heptatocellular carcinoma[J]. Bioinformatics, (2012), 28(24): 3191-3194.

[7] Su T, Wang W, Lv Z. Rapid Delaunay triangulation for randomly distributed point cloud data using adaptive Hilbert curve[J]. Computers \& Graphics, (2016), 54: 65-74.

[8] Guo C, Liu X, Jin M. The research on optimization of auto supply chain network robust model under macroeconomic fluctuations [J]. Chaos, Solitons \& Fractals, (2015).

[9] $\mathrm{Li} \mathrm{X}, \mathrm{Lv} \mathrm{Z}, \mathrm{Hu}$ J. XEarth: A 3D GIS Platform for managing massive city information[C]//Computational Intelligence and Virtual Environments for Measurement Systems and Applications (CIVEMSA), 2015 IEEE International Conference on. IEEE, (2015): 1-6.

[10] Yang J, Chen B, Zhou J. A Low-Power and Portable Biomedical Device for Respiratory Monitoring with a Stable Power Source[J]. Sensors, (2015), 15(8): 19618-19632.

[11] Guanqun Bao, Liang Mi, Yishuang Geng, Kaveh Pahlavan, A computer vision based speed estimation technique for localiz ing the wireless capsule endoscope inside small intestine, 36th Annual International Conference of the IEEE Engineering in Medicine and Biology Society (EMBC), Aug. (2014).

[12] Xinchao Song, Yishuang Geng, Distributed community detection optimization algorithm for complex networks, Journal of Networks, 9(10), 2758-2765, Jan. (2014).

[13] Zhang L, He B, Sun J. Double Image Multi-Encryption Algorithm Based on Fractional Chaotic Time Series[J]. Journal of Computational and Theoretical Nanoscience, (2015), 12: 1-7.

[14] Su T, Lv Z, Gao S. 3d seabed: 3d modeling and visualization platform for the seabed[C]. Multimedia and Expo Workshops (ICMEW), 2014 IEEE International Conference on. IEEE, (2014): 1-6.

[15] Yishuang Geng, Jin Chen, Ruijun Fu, Guanqun Bao, Kaveh Pahlavan, Enlighten wearable physiological monitoring systems: On-body rf characteristics based human motion classification using a support vector machine, IEEE transactions on mobile computing, 1(1), 1-15, Apr. (2015).

[16] Lv Z, Halawani A, Feng S. Multimodal hand and foot gesture interaction for handheld devices[J]. ACM Transactions on Multimedia Computing, Communications, and Applications (TOMM), (2014), 11(1s): 10.

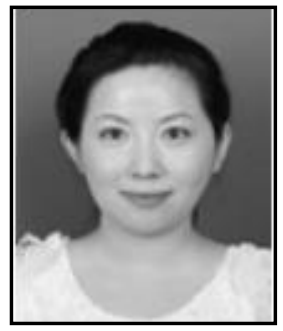

Xue Jia, She received her M.S. Degree in Industrial Economics from Xi'an university of Posts and Telecommunications in Xi'an, China. She is currently a lecturer in the Department of Health Management, Xi'an Medical University. Her research interest is mainly in the area of Industrial Economics, Health Economics . She has published more than ten research papers in scholarly journals in the above research areas and has participated in several books. 